Revue musicale OICRM

\title{
Processus d'apprentissage et de création des improvisateurs experts en musique classique
}

\section{Jean-Philippe Després}

Volume 4, numéro 1, 2017

Apprendre et enseigner la musique au XXI ${ }^{\mathrm{e}}$ siècle. Nouvelles propositions pédagogiques

URI : https://id.erudit.org/iderudit/1040300ar

DOI : https://doi.org/10.7202/1040300ar

Aller au sommaire du numéro

\section{Éditeur(s)}

Observatoire interdisciplinaire de création et recherche en musique (OICRM)

\section{ISSN}

2368-7061 (numérique)

Découvrir la revue

\section{Citer cet article}

Després, J.-P. (2017). Processus d'apprentissage et de création des improvisateurs experts en musique classique. Revue musicale OICRM, 4(1), 67-85. https://doi.org/10.7202/1040300ar
Résumé de l'article

Cet article présente une synthèse des principales contributions de mon projet de recherche doctorale qui porte sur l'improvisation musicale classique.Plus précisément, un devis de recherche en trois phases a été élaboré afin de documenter le parcours d'apprentissage, les stratégies de performances, ainsi que les approches d'enseignement-apprentissage d'instrumentistes et de pédagogues experts en improvisation musicale classique. Dans un premier temps, les résultats les plus significatifs de chacune des phases de la recherche sont présentés.Ensuite, les dimensions transversales aux trois phases sont discutées, avant de contextualiser les résultats par rapport au corpus de recherches théoriques et empiriques portant sur l'improvisation musicale. L'article se termine sur les principales implications pédagogiques de la recherche, celles qui ont émergé spécifiquement de chacune de ses phases, puis celles qui ressortent de l'analyse de l'ensemble des données. 


\title{
Processus d'apprentissage et de création des improvisateurs experts en musique classique
}

\author{
Jean-Philippe Després
}

\begin{abstract}
Résumé
Cet article présente une synthèse des principales contributions de mon projet de recherche doctorale qui porte sur l'improvisation musicale classique. Plus précisément, un devis de recherche en trois phases a été élaboré afin de documenter le parcours d'apprentissage, les stratégies de performances, ainsi que les approches d'enseignement-apprentissage d'instrumentistes et de pédagogues experts en improvisation musicale classique. Dans un premier temps, les résultats les plus significatifs de chacune des phases de la recherche sont présentés. Ensuite, les dimensions transversales aux trois phases sont discutées, avant de contextualiser les résultats par rapport au corpus de recherches théoriques et empiriques portant sur l'improvisation musicale. L'article se termine sur les principales implications pédagogiques de la recherche, celles qui ont émergé spécifiquement de chacune de ses phases, puis celles qui ressortent de l'analyse de l'ensemble des données.
\end{abstract}

Mots clés : développement del'expertise; enseignement-apprentissage ; improvisation; musique classique; stratégies.

\begin{abstract}
This article presents a synthesis of the main contributions of my doctoral research project on Western classical music improvisation. Specifically, a three-phase research design was developed to document the learning pathways, the performance strategies, and the teaching-learning approaches of expert instrumentalists and pedagogues in Western classical music improvisation. Firstly, the most significant results from each phase of the research are presented. Then, the transversal dimensions of the three phases are discussed, before contextualizing the results in relation to the corpus of theoretical and empirical studies on musical improvisation. The article concludes with the main pedagogical implications of the research, beginning with those that emerged specifically from each of its phases, then those that stood out of the analysis of the entire data set.
\end{abstract}

Keywords: expertise development; improvisation; strategies; teaching and learning; Western classical music. 
Dans un premier temps, cet article présente une synthèse des principales contributions de mon projet de recherche doctorale qui porte sur les processus d'apprentissage, de production et d'enseignement d'instrumentistes et de pédagogues experts en improvisation musicale classique ${ }^{1}$. Ensuite, il offre une perspective transversale sur les données récoltées, en intégrant les résultats des trois phases de la recherche pour en faire une analyse globale. Cette analyse a mené à la formulation de propositions pédagogiques originales basées sur l'ensemble de mon corpus de recherche.

En adoptant comme point de départ l'expérience vécue par des improvisateurs et des pédagogues experts, les résultats de chacune des trois phases de cette recherche ont offert une riche perspective sur la production et l'enseignement-apprentissage de l'improvisation musicale classique. En effet, mes résultats ont permis de mettre au jour une pluralité de parcours individuels ainsi que certains dénominateurs communs chez ces improvisateurs et pédagogues qui dédient une partie de leurs énergies, souvent à contre-courant, à la diffusion de l'improvisation, une pratique toujours marginale sur la scène et dans la pédagogie musicale classique. En plus de jeter un éclairage nouveau sur le parcours et la pratique de musiciens et de pédagogues reconnus comme étant des porteurs de la connaissance dans le domaine, l'analyse transversale des données offre de riches pistes pour encadrer l'enseignement-apprentissage de l'improvisation musicale classique. Alors que les recherches antérieures se sont intéressées principalement à l'improvisation musicale à l'orgue dans les contextes liturgiques et de concert (Chamblee 2008 ; Johansson 2008, 2011), ainsi qu'à l'improvisation de la période classique, au piano (Berkowitz 2009, 2010), cette recherche est la première à étudier le phénomène de l'improvisation chez les musiciens classiques selon une perspective plus globale, en incluant divers instruments et sans imposer de restrictions liées à une période ou à un courant historiques, afin de mieux représenter la pluralité et la complexité des pratiques improvisées appartenant au vaste domaine de la musique classique.

$\mathrm{Ma}$ recherche visait à documenter le parcours d'apprentissage, les stratégies de performances ainsi que les approches d'enseignement-apprentissage d'instrumentistes et de pédagogues experts en improvisation musicale classique. Ces trois dimensions m'ont mené à développer un devis méthodologique en trois phases. Dans un premier temps, je présente les résultats les plus significatifs de chacune des phases de ma recherche. Les dimensions transversales aux trois phases sont ensuite discutées avant de contextualiser les résultats par rapport au corpus de recherches théoriques et empiriques portant sur l'improvisation musicale, tout en soulignant les contributions spécifiques de ma recherche. L'article se termine sur les principales implications pédagogiques découlant de l'analyse transversale de mes résultats ainsi que sur diverses pistes qui pourraient être explorées dans le cadre de recherches ultérieures.

1 Ce projet de recherche a été approuvé par le Comité d'éthique de la recherche de l'Université Laval (CÉRUL), numéros de référence : 2013-110 et 2013-110 R1. 
La question de recherche suivante a été posée : "Quel parcours d'apprentissage a conduit les improvisateurs experts en musique classique à développer leurs compétences dans ce domaine? ». Afin de répondre à cette question, j'ai développé un dispositif méthodologique reposant sur une étude de cas multiple exploratoire. Plus précisément, j'ai mené des entrevues semi-dirigées à questions ouvertes par visiophonie auprès de huit improvisateurs experts en musique classique de la scène internationale, provenant de quatre pays différents, en adoptant une stratégie d'échantillonnage par choix raisonné homogène ${ }^{2}$. Ces entrevues étaient orientées autour du parcours d'apprentissage de l'improvisation des participants. Les résultats de cette phase ont offert un éclairage nouveau sur plusieurs dimensions du processus d'acquisition et de développement de l'expertise en improvisation musicale classique.

En effet, une analyse en profondeur des données récoltées lors de la phase 1 a révélé diverses composantes liées au " pourquoi » et au " comment » du processus d'apprentissage des experts en improvisation musicale classique, ce qui a permis de mettre au jour deux parcours d'apprentissage typiques et distinctifs : les improvisateurs natifs et immigrants. Alors que les improvisateurs natifs ont commencé à improviser dès les débuts de leur apprentissage musical, les improvisateurs immigrants ont commencé à improviser après plusieurs années de pratique instrumentale, généralement à la fin de leur parcours d'apprentissage formel ou en amorçant leur carrière professionnelle. Les improvisateurs natifs ont commencé à improviser spontanément, sans incitation extrinsèque apparente et ont développé leur habileté en improvisation spontanément, à l'image d'une langue maternelle. Les improvisateurs immigrants, pour leur part, ont entrepris l'apprentissage de l'improvisation pour tenter de combler une lacune ressentie dans leur parcours musical classique et ont acquis cette compétence d'une façon délibérée, telle une langue seconde. Divers facteurs ont motivé les improvisateurs immigrants interrogés à se consacrer à cette pratique, notamment le fait de percevoir l'improvisation comme étant un moyen de faire l'expérience : a) d'un retour à soi-même ; b) d'une rencontre humaine authentique ; c) d'un sentiment d'immédiateté lors du processus créatif ; et d) d'une expérience musicale égalitaire.

Enfin, les données ont également mis au jour un processus d'apprentissagedésapprentissage qui semble être sous-jacent à l'atteinte de l'expertise en improvisation, et ce, aussi bien chez les improvisateurs natifs qu'immigrants. Sur le plan pédagogique, les résultats laissent entrevoir deux recommandations principales. D'une part, l'habileté en improvisation peut être développée par une pratique assidue et délibérée, même à l'âge adulte. D'autre part, il est préférable d'intégrer l'improvisation dès l'amorce du processus d'apprentissage musical puisque son acquisition s'en trouve facilitée chez l'apprenant.

Les résultats concordent avec la position de divers auteurs (notamment Brophy 2001 ; Kenny et Gellrich 2002 ; Kratus 1995 ; Sloboda 1993) qui affirment

2 C'est-à-dire où le chercheur a utilisé certains critères afin de constituer un échantillon de cas relativement semblables ; ici des improvisateurs experts en musique classique. 
que la compétence en improvisation est acquise plutôt qu'innée. Plusieurs variables, telles que l'environnement socioculturel, la motivation, le dévouement, la propension à prendre des risques et les rencontres musicales exercent une plus grande influence sur le développement de l'expertise en improvisation que les attributs innés. Toutefois, les recherches antérieures ne font pas état du clivage existant entre les improvisateurs natifs et immigrants en musique classique. Les improvisateurs natifs et immigrants ont commencé à improviser dans des conditions tout à fait distinctes qui semblent avoir teinté leur relation avec l'improvisation ainsi que leur processus d'apprentissage. Ces deux parcours, fortement différenciés dans les débuts de l'apprentissage, tendent à s'estomper progressivement. En effet, que leurs compétences aient été développées " naturellement » ou qu'elles aient été acquises délibérément, le processus d'apprentissage des participants tend à être de plus en plus semblable au fur et à mesure qu'ils avancent sur la voie du développement de l'expertise en improvisation. Ce clivage entre improvisateurs natifs et immigrants n'a pas été identifié dans la littérature antérieure et ceci pourrait être expliqué par le fait que très peu de recherches ont étudié spécifiquement le processus d'apprentissage des improvisateurs experts en musique classique.

Un autre aspect important qui ressort de cette phase d'expérimentation est la grande valeur attribuée par les participants à l'immédiateté du processus créatif lors de l'improvisation. En effet, l'immédiateté a été identifiée par quatre participants comme étant l'une des raisons principales pour lesquelles ils valorisent l'improvisation au-delà des autres pratiques musicales. Cette immédiateté qui se manifeste par la création d'idées musicales en temps réel représenterait, selon la littérature, la caractéristique distinctive de l'improvisation (Ashley 2009 ; Csikszentmihalyi 1997 ; Johnson-Laird 2002 ; Kenny et Gellrich 2002).

Par ailleurs, le processus d'apprentissage-désapprentissage mis en lumière par six participants de la présente recherche peut être relié à diverses recherches antérieures en improvisation musicale classique. Premièrement, en ce qui a trait à la dimension « apprentissage ", Berkowitz $(2009,2010)$ et Johansson $(2008,2011)$ ont documenté l'apprentissage de l'improvisation qui est :

1. nourri dans le cadre d'une relation maître-apprenti (Johansson);

2. médiatisé par l'assimilation de connaissances déclaratives et procédurales (Berkowitz) ;

3. consolidé en utilisant les connaissances acquises lors de créations ex tempore (Berkowitz) et ;

4. raffiné lors de prestations devant public (Berkowitz et Johansson).

Bien que ces composantes fassent sans doute partie du processus d'apprentissage de l'improvisation, les résultats tendent à démontrer que cette liste ne serait ni exhaustive ni exclusive puisque deux improvisateurs natifs ont suivi un parcours d'apprentissage autodidacte et très peu systématique. Les résultats indiquent également que la partie "désapprentissage " du processus ne pourrait se manifester que subséquemment à l'assimilation préalable d'une grande quantité de connaissances et de compétences. Le désapprentissage ne correspond pas littéralement à l'oubli des connaissances, mais plutôt à leur transcendance ; en ce sens, ce processus semble être un facteur important pour permettre au musicien improvisateur d'expérimenter le flow (Després 
et al. 2016). En ce sens, l'improvisation se distingue de plusieurs autres domaines d'expertise par la combinaison complexe de processus qu'elle exige de celui qui la pratique (entre autres, la perception, l'écoute, le monitorage, le stockage en mémoire, le rappel, l'idéation, l'exécution et l'évaluation ; voir Johnson-Laird 2002 ; Kenny et Gellrich 2002 ; Lehmann, Sloboda et Woody 2007).

PHASE 2

Après avoir étudié, lors de la phase 1 de la recherche, le processus d'apprentissage des improvisateurs experts, je me suis intéressé à leur processus créatif lors de la phase 2. Afin d'étudier ce phénomène en profondeur, la question de recherche suivante a été formulée : "Quelles stratégies sont mises en œuvre par les improvisateurs experts en musique classique lors de leurs prestations ? " Pour répondre à cette question, j'ai mis en œuvre une stratégie de collecte de données centrée autour du protocole verbal rétrospectif avec aide à la remémoration subjective. Plus précisément, des rencontres individuelles ont été organisées avec cinq improvisateurs experts en musique classique internationalement reconnus. Lors de ces rencontres, j'ai d'abord invité le participant à improviser " comme il a l'habitude de le faire ». L'improvisation alors produite par le participant a été filmée à l'aide d'une minicaméra placée sur sa tête de telle sorte à capturer, de la manière la plus représentative possible, son point de vue subjectif.

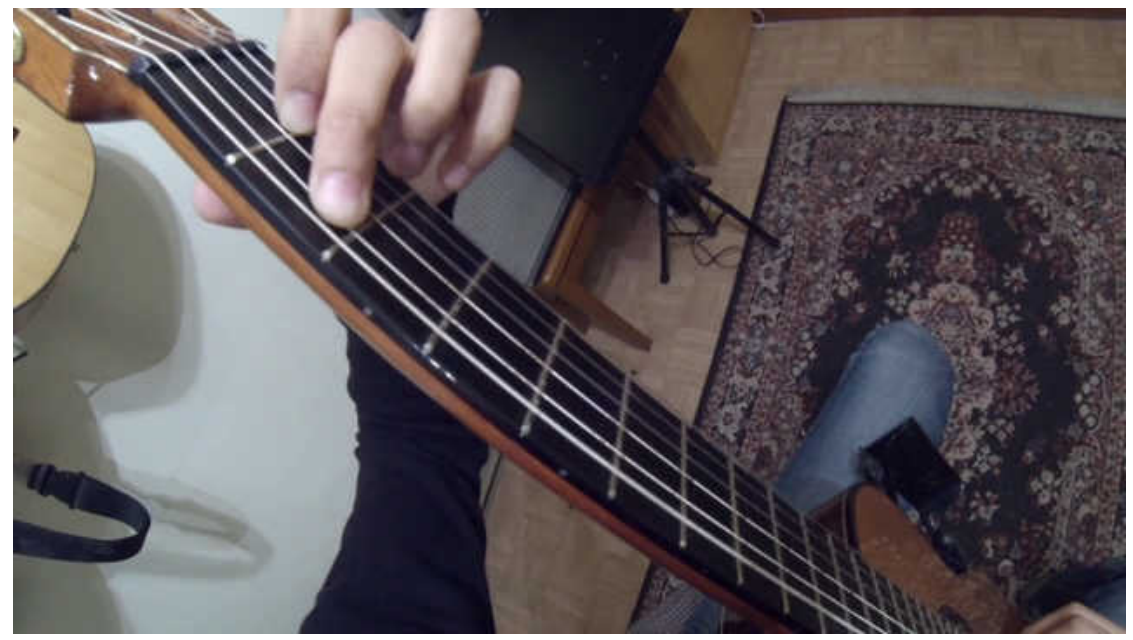

Extrait vidéo 1 : Exemple d'enregistrement $A / V$ d'une improvisation captée à l'aide d'une minicaméra portée sur la tête.

L'improvisation terminée, j'ai immédiatement mené un entretien semi-dirigé auprès $\mathrm{du}$ participant afin de récolter un protocole verbal rétrospectif de sa prestation en utilisant l'enregistrement audiovisuel de son improvisation comme aide à la remémoration. Lors de cet entretien, le participant a été invité à décrire son activité mentale lors de l'improvisation, mon objectif étant d'identifier les stratégies mises en œuvre lors de sa prestation. Après transcription, les entretiens ont été codés et analysés en utilisant une approche par catégorie mixte (c'est-à-dire combinant des catégories de codages inductives et déductives ; L'Écuyer 1987). Les catégories préexistantes étaient issues de la synthèse et de la mise à jour de revues de littératures publiées antérieurement 
(Dubé et Després 2012 ; Després et Dubé 2015). Tout au long du processus de codage, des catégories émergentes ont été créées et les catégories existantes ont été scindées ou fusionnées conséquemment à la compréhension dynamique et grandissante de mon objet d'étude ; les définitions respectives des catégories ont été revues et peaufinées conséquemment à ce processus.

Les résultats de la phase 2 présentent des contributions substantielles aux connaissances dans le domaine de la production experte en improvisation musicale classique. D'abord, cette phase a permis de mettre au jour 46 stratégies liées à la production de l'improvisation musicale classique. De plus, l'analyse de mes données a permis d'organiser les stratégies répertoriées en cinq catégories : de planification, conceptuelles, structurelles, atmosphériques et stylistiques ainsi qu'en temps réel. Cette structure permet de poser les fondements empiriques d'une nomenclature des stratégies de production de l'improvisation musicale classique. Cette catégorisation de niveau supérieur a émergé des données et n'était pas anticipée lors de l'élaboration du devis initial. Par ailleurs, le nombre et la variété des stratégies répertoriées, la diversité des points de mire employés (exemplifiée par les cinq catégories identifiées), ainsi que la moyenne de stratégies utilisées (moyenne $=24$ ) par les participants montrent que les improvisateurs experts en musique classique font appel à un vaste répertoire de stratégies et passent rapidement d'un mode de pensée à un autre lors d'une même improvisation.

Les résultats obtenus présentent à la fois d'importantes correspondances avec la littérature antérieure et des apports novateurs. D'une part, les stratégies d'improvisation identifiées dans la littérature antérieure ont toutes été utilisées par au moins un des participants de ma recherche, ce qui laisse entrevoir le fait que les improvisateurs experts en musique classique font effectivement appel aux stratégies présentées dans la littérature théorique (Ashley 2009 ; Clarke 1988 ; Kenny et Gellrich 2002 ; Pressing 1988) ou recensées dans les études empiriques menées dans le domaine du jazz (Berliner 1994 ; Hargreaves, Cork et Setton 1991 ; Norgaard 2008, 2011), de l'improvisation « libre » (Menezes 2010), de l'improvisation à l'orgue (Chamblee 2008 ; Johansson 2008, 2011) ou de l'improvisation dans la musique de la période classique (Berkowitz 2009, 2010). D'autre part, environ 61\% des stratégies identifiées dans la présente recherche n'avaient pas été documentées dans les études précédentes. Deux causes pourraient expliquer cet écart avec la littérature antérieure : 1) le choix méthodologique du protocole verbal rétrospectif avec aide à la remémoration subjective pourrait avoir permis d'accéder à des stratégies plus difficilement identifiables par l'intermédiaire d'autres stratégies de collecte de données ; et 2) les spécificités de la tâche d'improvisation classique solo exécutée par les participants de la présente recherche pourrait impliquer la mise en œuvre de stratégies différentes des types d'improvisation les plus fréquemment traités dans la littérature existante.

En plus de participer à l'avancement des connaissances dans le domaine spécifique de l'improvisation musicale classique, en révélant la diversité de stratégies utilisées par les experts du domaine, les contributions de la phase 2 de ma recherche se situent également sur le plan méthodologique. En effet, à partir de l'apport méthodologique de recherches récentes portant sur les stratégies des experts dans les domaines du sport (Mackenzie et Kerr 2012 ; Sparkes et Smith 2012 ; Williams et Ericsson 2005) et de la prise de décision (Omodei, McLennan et Wearing 2005), j’ai développé une 
méthode de collecte de données faisant appel au protocole verbal rétrospectif avec aide à la remémoration subjective afin de mettre au jour les stratégies mises en œuvre par les improvisateurs experts en musique classique. Cette décision méthodologique est fondée sur les contributions de divers auteurs. D'abord, van den Haak, De Jong et Schellens (2003) remarquent que le protocole verbal rétrospectif, comparativement au protocole simultané, est moins réactif et permet d'obtenir des verbalisations plus complètes. Ainsi, le protocole verbal rétrospectif représenterait une stratégie efficace pour étudier le processus créatif en profondeur, car il permet au chercheur d'accéder à la pensée privée d'individus créateurs sans imposer de limites à la durée de la collecte de données, a contrario du protocole verbal simultané, lors duquel les données sont collectées en temps réel lors de l'exécution de la tâche étudiée, ce qui peut limiter la richesse des données récoltées en plus d'interférer avec le processus créatif du participant. D'autre part, Hoc et Leplat (1983) ont démontré que le protocole verbal rétrospectif, s'il est accompagné d'une aide à la remémoration ${ }^{3}$, est mieux adapté que le protocole verbal simultané pour identifier les processus cognitifs mis en œuvre lors de l'exécution d'une tâche de résolution de problème. Pour cette remémoration, Rix et Biache (2004) préconisent l'utilisation d'une perspective subjective, laquelle est désormais facilitée par l'accessibilité des caméras miniatures de qualité qui peuvent être placées, par exemple, sur la tête de l'individu lorsqu'il effectue la tâche étudiée. Ce point de vue subjectiffavoriserait un processus de remémoration plus valide qu'une perspective objective, c'est-à-dire qu'il serait en plus grande adéquation avec la réalité vécue de l'individu. Par ailleurs, alors que quelques recherches antérieures ont documenté l'activité mentale d'improvisateurs experts à l'aide du protocole verbal rétrospectif dans les contextes du jazz (Hargreaves, Cork et Setton 1991 ; Mendonça et Wallace 2004 ; Norgaard 2008) et de l'improvisation " libre »(Menezes 2010), à ce jour aucune recherche n'a utilisé cette stratégie de collecte de données dans le contexte de l'improvisation musicale classique. De plus, ma recherche serait la première à utiliser le protocole verbal rétrospectif avec aide à la remémoration subjective pour étudier l'activité mentale de musiciens, et ce, pour tous genres et toutes pratiques confondus. Selon les propos spontanément exprimés par les participants, l'utilisation du protocole verbal rétrospectif avec aide à la remémoration subjective s'est avérée une méthode efficace pour les replonger dans leur expérience vécue lors de l'improvisation, ce qui représente une condition nécessaire à la validité interne des données lorsqu'elles sont récoltées lors d'une verbalisation post hoc. De surcroît, la richesse des données auxquelles cette stratégie de collecte de données m'a permis d'accéder laisse entrevoir qu'il s'agit d'une approche méthodologique qui revêt un fort potentiel pour étudier l'activité mentale liée à l'exécution d'une tâche, particulièrement lorsque la verbalisation simultanée risque de s'avérer trop réactive.

3 Par exemple, si le participant visionne un enregistrement de son processus de résolution de problème alors qu'il produit le protocole verbal rétrospectif. 
PhASE 3

La troisième phase de la recherche portait plus particulièrement sur les processus d'enseignement-apprentissage des improvisateurs et pédagogues experts en improvisation musicale classique. Cette phase visait à répondre à la question suivante : "Quelles caractéristiques du parcours d'apprentissage ou de la pratique pédagogique des experts du domaine pourraient contribuer à bonifier l'enseignement-apprentissage de l'improvisation musicale classique? ». Pour répondre à cette question, deux objectifs ont été formulés :

1. répertorier les recommandations pédagogiques et les stratégies d'enseignement-apprentissage de musiciens et de pédagogues experts en improvisation musicale classique;

2. structurer les éléments répertoriés afin de formuler une proposition pédagogique cohérente.

Afin d'atteindre ces objectifs, l'échantillon a été bonifié par la participation d'experts en enseignement de l'improvisation musicale classique. Quinze participants ont été interviewés, dont quatre improvisateurs experts et deux pédagogues experts ; ces deux dernières catégories n'étant pas mutuellement exclusives, neuf des participants sont à la fois improvisateurs et pédagogues experts. Par ailleurs, sept participants étaient originaires du Canada, cinq de la France, un d'Israël, un de l'Allemagne et un du Mexique ; dix entrevues se sont déroulées par visiophonie et cinq dans le cadre d'entrevues individuelles. L'analyse de des données m'a permis de poser les bases d'une proposition pédagogique de l'enseignement-apprentissage de l'improvisation musicale classique fondée sur des données empiriques.

En portant mon regard sur les stratégies qui ont permis aux improvisateurs experts de développer leur habileté en improvisation, ainsi que sur les stratégies qui sont mises en œuvre par les pédagogues experts en improvisation musicale classique lors de leur enseignement, j'ai identifié diverses composantes liées à l'enseignement-apprentissage de cette compétence complexe qu'est l'improvisation, que j'ai regroupées sous deux axes : 1) compétences à développer chez l'apprenant improvisateur, et 2) approches d'enseignement-apprentissage de l'improvisation musicale classique. Tout d'abord, les participants ont mentionné 18 compétences qui, à leur avis, devaient être développées par l'apprenant improvisateur et qui influenceraient favorablement le développement de ses habiletés d'improvisation. J'ai regroupé ces compétences en trois grandes catégories : 1) musicales et théoriques, 2) synchrones et 3) autodidactiques. Finalement, 23 approches d'enseignement-apprentissage - des propositions d'activités concrètes visant à développer les habiletés d'improvisation de l'apprenant - ont été identifiées. Ces approches d'enseignement-apprentissage ont été regroupées en sept catégories : 1) fondamentales, 2) planificatrices, 3) sonores, 4) conceptuelles, 5) structurelles, 6) stylistiques et 7) contextuelles.

Les compétences autodidactiques et synchrones identifiées chez les participants de la présente recherche viennent étoffer les connaissances antérieures liées au volet psychologique de l'improvisation. En plus de la capacité de prendre des risques et de savoir accueillir la fausse note - deux compétences dont l'importance est abondamment soulignée dans les recherches antérieures (Dubé et Després 2012) -, mes résultats 
mettent en valeur d'autres compétences qui sont moins fréquemment abordées dans la littérature telles que : faire preuve de "présence " et d'ouverture, développer sa capacité d'adaptation et favoriser la concertation entre l'instinct et le savoir-faire. La primauté accordée aux compétences synchrones chez les participants de la présente étude pourrait être expliquée par le fait que ces musiciens, de par leur formation classique, définissent leur rapport à l'improvisation en l'opposant à l'interprétation - pratique sur laquelle l'accent est généralement mis dans la pédagogie musicale classique traditionnelle -, où la quête de la perfection est généralement véhiculée. En effet, selon les propos des participants l'improvisation et l'interprétation se traduisent par un rapport à l'inattendu et aux « erreurs » qui est complètement différent. Alors que le musicien improvisateur vise à développer sa capacité à intégrer musicalement l'inattendu et les éventuelles " erreurs » à ses prestations, l'interprète recherche ordinairement plutôt à offrir une prestation exempte d'erreurs, d'accrocs techniques ou d'évènements inattendus.

Par ailleurs, il appert qu'une grande majorité des compétences répertoriées seraient transversales aux différents genres musicaux où l'improvisation est pratiquée. Néanmoins, certaines compétences seraient plus spécifiques à certains genres en raison des modalités d'apprentissage prédominantes et des caractéristiques du langage propres à chaque genre musical improvisé. Finalement, alors que certaines approches d'enseignement-apprentissage répertoriées sont évoquées dans la littérature antérieure (notamment par Kenny et Gellrich 2002 et Thompson et Lehmann 2004), la liste proposée ici est plus exhaustive et comporte certaines approches moins usuelles telles que : travailler la laideur et la beauté du son, jouer en déplaçant des accents ${ }^{4}$, s'ancrer sur tonique-dominante, ériger la structure en temps réel, faire de l'improvisation générative et cultiver le sentiment d'urgence. Ces composantes offrent à celui qui s'intéresse à l'improvisation - qu'il soit enseignant ou apprenant, de niveau novice, débutant ou intermédiaire des balises pour guider son apprentissage instrumental ou sa pratique pédagogique. Ainsi, la troisième phase de ma recherche permet de poser les fondations empiriques nécessaires au développement de matériel pour l'enseignement-apprentissage de l'improvisation musicale classique destiné à être utilisé aux niveaux préuniversitaire et universitaire. De plus, en continuité avec les conclusions de la phase 2 de la recherchequi ont montré que les improvisateurs experts mettent en œuvre une grande variété de stratégies lors de l'improvisation -, les résultats de la phase 3 mettent en valeur le fait qu'une pédagogie de l'improvisation devrait aller au-delà des considérations liées à la sélection des notes à jouer; elle devrait être holistique et toucher aux diverses facettes de l'improvisation, de sa planification à l'établissement de sa structure formelle.

\section{SYNTHÈSE}

En résumé, les trois phases de mon projet doctoral ont contribué à enrichir les connaissances au sujet de l'enseignement-apprentissage et de la production de l'improvisation classique, posant ainsi les fondements empiriques d'une pédagogie efficiente 
de l'improvisation musicale classique. À ce jour, il s'agit probablement de la recherche la plus substantielle portant sur les processus d'apprentissage, de production et d'enseignement de l'improvisation musicale classique, en documentant ces phénomènes à partir du point de vue des acteurs qui sont les porteurs de l'expertise dans ce domaine.

\section{IMPLICATIONS PÉDAGOGIQUES}

Les résultats obtenus au fil des trois phases de l'expérimentation laissent entrevoir diverses implications liées à l'enseignement-apprentissage de l'improvisation musicale classique. Dans un premier temps, je présenterai les considérations générales qui ressortent de chacune des phases de la recherche. Ensuite, j'aborderai le corpus de manière transversale en présentant l'ensemble des savoir-être, savoir-faire et savoirs à développer chez le musicien improvisateur, ainsi qu'une synthèse des recommandations pédagogiques qui ont été mises de l'avant par les participants dans l'ensemble des entrevues qui ont été menées.

Phase 1 : intégrer l'improvisation dès le début

Mes résultats suggèrent que l'introduction de l'improvisation aux débuts de l'apprentissage musical serait grandement bénéfique pour l'apprenant. Alors que tous les experts interrogés considéraient l'improvisation comme étant une pratique au potentiel libérateur et créatif inégalé, il appert que les improvisateurs natifs perçoivent leur apprentissage comme étant un processus moins laborieux que les improvisateurs immigrants. En effet, le parcours d'apprentissage des improvisateurs natifs - qui ont appris d'abord à écouter et à "parler " la musique avant de la lire et de l'écrire évoque l'apprentissage « naturel » des jeunes qui assimilent leur langue maternelle par immersion culturelle et la maîtrisent avec une grande fluidité.

Phase 2 : varier les stratégies d'improvisation et passer d'une stratégie à l'autre

Les principales implications pédagogiques découlant de la phase 2 de la recherche sont les suivantes : les apprenants devraient être guidés dans le développement des capacités : 1) de mettre en œuvre un répertoire de stratégies variées, agissant à divers niveaux (planification, conceptuel, structurel, atmosphérique et stylistique, et en temps réel) ; et 2) de passer d'une stratégie à une autre tout en sélectionnant les stratégies qui sont les mieux adaptées aux circonstances musicales et interactionnelles ponctuelles de l'improvisation.

\section{Phase 3 : adopter une approche pédagogique plurielle}

Reflétant la pluralité des stratégies pouvant être mises en œuvre lors de l'improvisation qui a été identifiée lors de la phase 2, les résultats de la phase 3 suggèrent que la compétence en improvisation devrait être nourrie par une grande variété de stratégies d'enseignement-apprentissage. Ces stratégies d'enseignement-apprentissage devraient comprendre des activités de fondamentales (en marge de l'improvisation), planificatrices (avant l'improvisation), ainsi que d'établissement des dimensions sonores, conceptuelles, structurelles, stylistiques et contextuelles de l'improvisation. 
Propositions pédagogiques transversales

Les tableaux proposés en annexe présentent respectivement les savoir-être, savoir-faire et savoirs impliqués lors de l'apprentissage et de la création de l'improvisation et les propositions pédagogiques qui ont été rencontrées lors des trois phases ${ }^{5}$ de la recherche. Sans constituer un modèle pédagogique en soi, ces données représentent néanmoins des balises fondées sur des données empiriques permettant de guider l'enseignant ou l'apprenant qui souhaiterait intégrer l'improvisation à sa pratique ou encore parfaire ses compétences en improvisation.

En somme, les savoir-être, savoir-faire et savoirs liés à l'apprentissage et à la création de l'improvisation musicale peuvent être acquis et renforcés par diverses stratégies, lesquelles peuvent être regroupées en quatre catégories :

1. fondamentales : cibler les apprentissages et les travailler isolément lors de répétitions ;

2. contextuelles : établir les conditions générales du contexte créatif de l'improvisation ;

3. planificatrices: déterminer certaines caractéristiques musicales spécifiques de l'improvisation ;

4. performatives: idéer ou sélectionner en temps réel au cours de l'improvisation, certaines composantes du discours musical.

L'intégration de stratégies variées, appartenant à chacune de ces quatre catégories lors de l'enseignement-apprentissage de l'improvisation contribuerait à développer les compétences de l'apprenant de façon holistique. Finalement, considérant la relative importance qu'occupent les recommandations liées à l'apprentissage de l'improvisation par l'improvisation pour les participants de la présente recherche, il serait intéressant d'expérimenter une approche d'enseignement-apprentissage principalement médiatisée par des activités réelles, sinon tout au moins réalistes, plutôt que par un contexte de répétition plus traditionnel, isolé de la prestation et plus linéaire.

\section{CONCLUSION}

Malgré l'intérêt croissant qui lui est accordé dans les programmes pédagogiques, sur la scène et dans la recherche scientifique, l'improvisation tarde toujours à s'imposer dans la pratique pédagogique en musique. Les résultats présentés ci-haut offrent des bases empiriques solides sur lesquelles édifier une pédagogie de l'improvisation musicale classique. Le processus de mise en œuvre des stratégies proposées, les obstacles rencontrés ainsi que leurs effets sur l'aisance en improvisation et sur les autres habiletés musicales et sociales de l'apprenant constituent des objets études centraux pour la poursuite de la recherche dans ce domaine qui devraient tenir compte des limitations relevées dans le cadre de la présente recherche.

5 Dans les tableaux, les numéros entre parenthèses indiquent ainsi la phase de la recherche lors de laquelle les savoir-être, savoir-faire, savoirs et propositions pédagogiques présentés ont été identifiés. 


\section{Limitations}

Les principales limites de ce projet sont liées au nombre de participants et à la représentativité des instruments. La taille relativement réduite de l'échantillon ne permet pas d'offrir une vision généralisable des processus d'apprentissage, d'enseignement et de production des improvisateurs et pédagogues experts en musique classique. De plus, elle ne permet pas? de comparaisons entre les divers instruments représentés dans mon étude. Il est ainsi envisageable que certaines caractéristiques liées à l'apprentissage ou à la production de la musique improvisée spécifiques à certains instruments ne soient pas représentées par mes résultats ; on peut, par exemple, penser à celles qui seraient propres aux instruments harmoniques ou monodiques.

Par ailleurs, la phase 2 présente certaines limitations inhérentes à la stratégie de collecte de données utilisée. Le protocole verbal rétrospectif ne permet pas d'accéder aux mécanismes cognitifs inconscients du participant et est susceptible de faire place à certains biais. La verbalisation des processus cognitifs liés à l'improvisation est une tâche qui, en soi, est susceptible de représenter une grande difficulté pour le participant puisque ces processus peuvent être en partie inconscients ou automatisés et qu'ils ne reposent pas forcément sur la logique (Van Someren, Barnard et Sandberg 1994). Ainsi, le protocole verbal peut entraîner des distorsions entre la réalité et les propos du participant puisqu'il exige à ce dernier de verbaliser de façon cohérente et linaire un processus complexe et possiblement non linéaire. Par exemple, le participant est susceptible de donner a posteriori plus de cohérence à ses actions qu'elles n'en avaient alors qu'il réalisait la tâche. Par ailleurs, des biais peuvent également être induits, par exemple, par l'oubli ou par l'omission volontaire d'informations par le participant (ibid.). Malgré les limites qui lui sont associées, en considérant les questions, les objectifs et le caractère exploratoire de ma recherche, ainsi que la rareté relative des improvisateurs experts en musique classique, le protocole verbal rétrospectif demeure l'outil de collecte de données qui s'avérait le mieux adapté à mon objet d'étude. En effet, les qualités de mon approche méthodologique qualitative fondée sur l'étude de cas, les entrevues semi-dirigées à questions ouvertes et un échantillon relativement réduit surpassent ses limitations ; la grande richesse des données recueillies en témoigne.

\section{Pistes pour les recherches ultérieures}

Ma recherche a permis d'identifier des pistes de recherches intéressantes liées à l'enseignement-apprentissage et à la production de l'improvisation musicale classique.

Avant tout, en ce qui a trait à l'enseignement-apprentissage de l'improvisation, l'observation directe des répétitions de musiciens experts en improvisation reconnus permettrait d'enrichir notre compréhension du comment de l'apprentissage de l'improvisation et du développement de l'expertise dans ce domaine. Ensuite, il faudrait mesurer l'effet d'une pédagogie de l'improvisation fondée sur les propositions pédagogiques récoltées lors de la phase 3 de la recherche sur le développement : 1) de la compétence en improvisation de l'apprenant et 2) de ses autres habiletés musicales et non musicales. Finalement, le dispositif méthodologique du protocole verbal rétrospectif avec aide à la remémoration subjective pourrait être utilisé dans un contexte pédagogique 
afin de documenter le processus d'apprentissage, de mise en œuvre et les effets des différentes stratégies d'improvisation recensées en contexte réel auprès d'apprenants de divers niveaux, jouant divers instruments.

Par ailleurs, afin de poursuivre la recherche sur le processus de production de l'improvisation musicale classique, il s'avérerait essentiel de répliquer l'approche méthodologique utilisée dans le cadre de la phase 2 auprès d'un plus grand nombre de participants en vue de détailler, notamment, les variations qui pourraient exister d'un instrument à l'autre ou d'un genre improvisé à l'autre. Par ailleurs, l'étape suivante serait de documenter le processus de production de l'improvisation musicale classique dans un environnement naturel. Ainsi, la stratégie de collecte de données du protocole verbal rétrospectif avec aide à la remémoration subjective pourrait être mise en œuvre lors de concerts devant public afin de documenter la réalité du musicien improvisateur sur la scène en tenant compte des facteurs liés à ce contexte particulier, tels que les interactions avec le public ou l'éventuelle adrénaline du moment. De plus, ma stratégie de collecte de données pourrait être utilisée simultanément auprès des différents membres d'un ensemble qui improvise en concert. Finalement, dans tous les cas, il serait pertinent de faire analyse des données vidéo récoltées selon une perspective subjective lors de l'improvisation. En effet, en observant ces enregistrements, il m'est apparu évident que divers éléments, tels que les mouvements de tête du musicien, sa gestuelle, ses hésitations et sa technique, étaient potentiellement de riches porteurs de sens. À titre exploratoire, ces données pourraient être mises en parallèle avec les propos du musicien pour analyser les rapports entre les mouvements corporels et l'expérience vécue du musicien improvisateur, par exemple pour identifier les variations d'états rapportées par le musicien lorsque son corps est fixe versus lorsqu'il est en mouvement.

\section{BIBLIOGRAPHIE}

Ashley, Richard (2009), " Musical Improvisation », dans Susan Hallam, Ian Cross et Michael Thaut (dir.), The Oxford Handbook of Music Psychology, New York, NY, Oxford University Press, p. 413420.

Berkowitz, Aaron (2009), "Cognition in Improvisation. The Art and Science of Spontaneous Musical Performance ", Thèse de doctorat, Harvard University.

Berkowitz, Aaron (2010), The Improvising Mind. Cognition and Creativity in the Musical Moment, Oxford, Oxford University Press.

Berliner, Paul F. (1994), Thinking in Jazz. The Infinite Art of Improvisation, Chicago, University of Chicago Press.

Brophy, Timothy S. (2001) «Developing Improvisation in General Music Classes », Music Educators Journal, $n^{\circ} 88$, p. 34-53.

Chamblee, Catherine B. (2008), "Cognitive Processes of Improvisation. Performers and Listeners in the Organ Tradition and Contemporary Gospel Styles ", Thèse de doctorat, University of Pennsylvania.

Clarke, Eric F. (1988), "Generative Principles in Music Performance », dans John A. Sloboda (dir.), Generative Processes in Music. The Psychology of Performance Improvisation and Composition, Oxford, Clarendon Press, p. 1-26. 
Csikszentmihalyi, Mihaly (1997), Creativity. Flow and the Psychology of Discovery and Invention, New York, Harper perennial.

Després, Jean-Philippe, et Francis Dubé, (2015), « Revue de littérature des écrits scientifiques portant sur l'improvisation musicale. Identification des concepts clés et des recommandations pédagogiques liés à ce domaine ", Les Cahiers de la Société québécoise de recherche en musique, vol. 15, n 1, p. 5566.

Després, Jean-Philippe, et al. (2016) " Expert Improvisers in Western Classical Music Learning Pathways ", Thinking Skills and Creativity, vol. 22, p. 167-179.

Dubé, Francis, et Jean-Philippe Després (2012), « Proposition d'un cadre conceptuel pour aider le professeur d'instrument à intégrer l'improvisation musicale à son acte pédagogique ", Intersections. Canadian Journal of Music, vol. 32, no 1-2, p. 143-165, http://id.erudit.org/iderudit/1018582ar, consulté le 20 mars 2017.

Gordon, Edwin E. (1997). Learning Sequences in Music. Skill, Content and Patterns; A Music Learning Theory, Chicago, GIA Publications.

Haak, Maaike van den, De Jong, Menno, et Peter Jan Schellens (2003), «Retrospective vs. Concurrent Think-Aloud Protocols. Testing the Usability of an Online Library Catalogue », Behaviour \& Information Technology, vol. 22, n ${ }^{\circ}$ 5, p. 339-351.

Hargreaves, David J., Cork, Conrad A., et Tina Setton (1991), " Cognitive Strategies in Jazz Improvisation. An Exploratory Study ", Canadian Journal of Research in Music Education, vol. 33, $\mathrm{n}^{\circ} 1, \mathrm{p} .47-54$.

Hoc, Jean Michel, et Jacques Leplat (1983), « Evaluation of Different Modalities of Verbalization in a Sorting Task », International Journal of Man-Machine Studies, vol. 18 n 3, p. 283-306.

Johansson, Karin (2008), « Organ Improvisation-Activity, Action and Rhetorical Practice », Thèse de doctorat, Lund University.

Johansson, Karin (2011), « Organ Improvisation. Edition, Extemporization, Expansion, and Instant Composition ", dans David Hargreaves, Dorothy Miell et Raymond MacDonald (dir.), Musical Imaginations. Multidisciplinary Perspectives on Creativity, Performance and Perception, Oxford, Oxford University Press, p. 220-232.

Johnson-Laird, Philip N. (2002), « How Jazz Musicians Improvise », Music Perception, vol. $19 \mathrm{n}^{\circ} 3$, p. $415-442$.

Kenny, Barry J., et Martin Gellrich (2002), «Improvisation », dans Richard Parncutt et Gary McPherson (dir.), The Science and Psychology of Music Performance, New York, Oxford University Press, p. 117134.

Kratus, John (1995), « A Developmental Approach to Teaching Music Improvisation », International Journal of Music Education, vol. 26, nº 1, p. 27-38.

L'Écuyer, René (1987), «L'analyse de contenu. Notion et étapes », dans Jean-Pierre Deslauriers (dir.), Les méthodes de la recherche qualitative, Québec, Les Presses de l'Université du Québec, p. 49-65.

Lehmann, Andreas C., Sloboda, John A., et Robert H. Woody (2007), " Composition and Improvisation ", dans Psychology for Musicians. Understanding and Acquiring the Skills, New York, Oxford University Press, p. 127-144.

Mackenzie, Susan Houge, et John H. Kerr (2012), « Head-Mounted Cameras and Stimulated Recall in Qualitative Sport Research ", Qualitative Research in Sport, Exercise and Health, vol. 4, n 1,p. 5161.

Mendonça, David, et William A. Wallace (2004), « Cognition in Jazz Improvisation. An Exploratory Study ", 26th Annual Meeting of the Cognitive Science Society, Chicago, http://escholarship.org/uc/ item/46v6s1qk, consulté le 15 mai 2012.

Menezes, José Manuel Amaro (2010), "Creative Process in Free Improvisation ", Mémoire de maîtrise, University of Sheffield, http://home.uevora.pt/ jmenezes/jmdissertation.pdf, consulté le 2 juin 2012. 
Norgaard, Martin (2008), «Descriptions of Improvisational Thinking by Artist-Level Jazz Musicians», Thèse de doctorat, University of Texas, https://repositories.lib.utexas.edu/handle/2152/17893, consulté le 30 mars 2012.

Norgaard, Martin (2011), «Descriptions of Improvisational Thinking by Artist-Level Jazz Musicians », Journal of Research in Music Education, vol. 59, n 2, p. 109-127.

Omodei, Mary M., McLennan, Jim, et Alexander J. Wearing (2005) « How Expertise Is Applied in Real-World Dynamic Environments. Head Mounted Video and Cued Recall as a Methodology for Studying Routines of Decision Making ", dans Betsch Tilmann et Haberstroh Susanne (dir.), The Routines of Decision Making, Mahwah, Lawrence Erlbaum Associates Publishers, p. 271288.

Pressing, Jeff (1988), «Improvisation. Methods and Models », dans John A. Sloboda (dir.), Generative Processes in Music, Oxford, Oxford University Press, p. 129-178.

Rix, Géraldine, et Marie-Joseph Biache (2004), « Enregistrement en perspective subjective située et entretien en re situ subjectif. Une méthodologie de constitution de l'expérience ", Intellectica, vol. 38, p. 363-396.

Sloboda, John (1993), « Musical Ability », dans Gregory R. Bock Organizer et Kate Ackrill (dir.), Ciba Foundation Symposium 178 - The Origins and Development of High Ability, John Wiley \& Sons, Ltd, p. 106-118.

Sparkes, Andrew C., et Brett Smith (2012), " Embodied Research Methodologies and Seeking the Senses in Sport and Physical Culture. A Fleshing out of Problems and Possibilities ", Research in the Sociology of Sport, vol. 6, p. 167-190.

Thompson, Sam, et Andreas C. Lehmann (2004), « Strategies for Sight-Reading and Improvising Music ", dans Aaron Williamon (dir.), Musical Excellence. Strategies and Techniques to Enhance Performance, Oxford, Oxford University Press, p. 143-159.

Van Someren, Maarten W., Barnard, Yvonne F., et Jacobijn A. C. Sandberg (1994), The Think Aloud Method. A Practical Guide to Modelling Cognitive Processes, London, Academic Press.

Williams, A. Mark, et K. Anders Ericsson (2005), " Perceptual-Cognitive Expertise in Sport. Some Considerations When Applying the Expert Performance Approach ", Human Movement Science, vol. $24, \mathrm{n}^{\circ} 3$, p. 283-307. 
ANNEXE A : Savoir-être, savoir-faire et savoirs impliqués lors de l'apprentissage et de la création de l'improvisation.

\begin{tabular}{|c|c|c|}
\hline & & Description \\
\hline \multirow{7}{*}{ 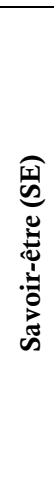 } & $\begin{array}{l}\text { Autonomie et autodidaxie } \\
(1,3)\end{array}$ & Être curieux, motivé à apprendre par soi-même et outillé pour le faire. \\
\hline & Interactions (1) & $\begin{array}{l}\text { Savoir communiquer de manière non verbale avec le public ou les autres } \\
\text { musiciens. Par exemple, ressentir ou anticiper leurs réactions et agir en } \\
\text { conséquence. }\end{array}$ \\
\hline & Prise de risques $(1,3)$ & $\begin{array}{l}\text { Faire preuve d'ouverture, dire « oui » à toutes les occasions d'improviser, } \\
\text { accepter de sortir de ses habitudes et développer sa capacité d'adaptation à } \\
\text { l'inconnu. }\end{array}$ \\
\hline & Auto-efficacité $(1,3)$ & Croire en son propre potentiel à improviser, éviter l'autocritique, être confiant. \\
\hline & Embodiment $(1,3)$ & Être présent, «bien dans son corps », lors de l'improvisation. \\
\hline & $\begin{array}{l}\text { Instinct vs savoir-faire }(1, \\
3)\end{array}$ & $\begin{array}{l}\text { Avoir la capacité de passer avec fluidité d'un mode de pensée rationnel à un } \\
\text { mode de pensée instinctif (et vice-versa) lors de l'improvisation. }\end{array}$ \\
\hline & $\begin{array}{l}\text { Monitoring et auto- } \\
\text { évaluation réflexive (3) }\end{array}$ & $\begin{array}{l}\text { Faire la surveillance du résultat sonore de ses actions lors de l'improvisation, } \\
\text { pour ensuite s'évaluer et s'adapter, si nécessaire, en fonction de cette évaluation. }\end{array}$ \\
\hline \multirow{8}{*}{ 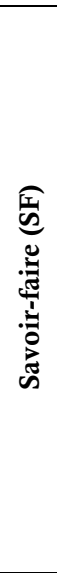 } & Maîtrise technique (2) & $\begin{array}{l}\text { Posséder le niveau de technique instrumentale nécessaire pour réaliser ses idées } \\
\text { musicales. }\end{array}$ \\
\hline & Audiation $^{*}(3)$ & Entendre intérieurement ses idées musicales avant de les jouer. \\
\hline & Adéquation technique $^{\dagger}(1)$ & $\begin{array}{l}\text { Développer une technique instrumentale souple, adaptée à l'instantanéité du } \\
\text { processus créatif improvisé. }\end{array}$ \\
\hline & $\begin{array}{l}\text { Transfert des } \\
\text { connaissances }(1,3)\end{array}$ & $\begin{array}{l}\text { Appliquer les connaissances apprises dans un contexte musical donné dans un } \\
\text { autre contexte, par exemple, appliquer les techniques d'analyse jazz en musique } \\
\text { classique. }\end{array}$ \\
\hline & Planification $(1,2)$ & $\begin{array}{l}\text { Anticiper certaines dimensions de l'improvisation avant de commencer à jouer, } \\
\text { afin d'établir un cadre qui, bien souvent, ouvre plutôt que limite les possibilités } \\
\text { créatives. }\end{array}$ \\
\hline & Banque d'idées (2) & Intégrer des idées musicales pré-mémorisées à son improvisation. \\
\hline & Développement (2) & $\begin{array}{l}\text { Varier et permuter les idées provenant de sa banque d'idées ou celles qui ont été } \\
\text { jouées précédemment au cours de l'improvisation. }\end{array}$ \\
\hline & Désapprentissage (1) & $\begin{array}{l}\text { Lors de l'improvisation, mettre ponctuellement en veilleuse ses connaissances } \\
\text { théoriques }{ }^{\ddagger} \text {. }\end{array}$ \\
\hline \multirow{10}{*}{ 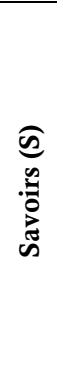 } & Rythmiques (2) & Figures rythmiques, tempo et des métriques. \\
\hline & Mélodiques $(2,3)$ & Gammes, modes et arpèges. \\
\hline & Harmoniques $(2,3)$ & Accords et structures harmoniques. \\
\hline & Contrapuntiques (3) & Notions d'écriture contrapuntiques. \\
\hline & Tonals (2) & Tonalités, modulations et fonctions des degrés mélodiques. \\
\hline & Motiviques et thématiques & Motifs et thèmes du répertoire ou inventés par le musicien. \\
\hline & Formels (2) & Principes d'organisation structurelle et formes musicales. \\
\hline & Atmosphériques (2) & Relation entre les émotions et la musique. \\
\hline & Stylistiques (2) & Euvres, genres, styles et compositeurs. \\
\hline & Timbrals (2) & Registre, timbre, sonorités et techniques étendues des instruments. \\
\hline
\end{tabular}

* L'audiation est un terme créé par Gordon (1997) pour dénommer l'aptitude à entendre intérieurement les sons et à leurs donner un sens.

$\dagger \quad$ La technique correspond ici à l'ensemble des habiletés physiques qui permettent de réaliser ses idées musicales sur le plan sonore.

$\ddagger \quad$ Certains musiciens le font spontanément, mais d'autres ont plus de difficulté à mettre de côté les notions musicales (notes, gammes, accords, tonalité) lors de l'improvisation. Alors que ces balises ont souvent une valeur inestimable pour le musicien improvisateur, elles peuvent également limiter sa créativité s'il n'est pas en mesure de les "oublier» temporairement pour les transcender. 
ANNEXE B : Propositions pédagogiques rencontrées lors des trois phases de la recherche, et savoirs, savoir-faire et savoir-être principalement engagés pour chaque proposition.

Propositions pédagogiques

Fondamentales (lors des répétitions personnelles)

Écouter les grands improvisateurs (3)

En enregistrement ou en concert.

Repiquer (3)

Des motifs, des solos, des sections de pièces ou des pièces complètes.

Construire sa banque d'idées $(2,3)$

Noter ou enregistrer des idées musicales inventées, tirées du répertoire ou customisées.

Transposer, séquencer, varier ou ornementer $(2,3)$

Des motifs, des phrases, des thèmes ou des pièces provenant du répertoire ou de sa banque d'idées.

Jouer en déplaçant des accents (3)

Par exemple, jouer du répertoire ou des improvisations classiques avec des appuis « jazz », sur les temps 2 et 4 , pour améliorer le contrôle rythmique de l'apprenant.

Explorer les limites de son instrument (3)

Travailler la sonorité de l'instrument en expérimentant les timbres extrêmes, qui sont souvent évités lors du travail d'interprétation, pour mieux connaître les possibilités et limites de son instrument et de dépasser la peur de faire un son « laid».

Contextuelles (définir les conditions entourant l'improvisation)

Cultiver le sentiment d'urgence (3)
Préparer une prestation en un court laps de temps, par exemple monter de toutes pièces un concert qui sera présenté la journée même ou dans la semaine.

Faire de l'improvisation interdisciplinaire (3)

Improviser en collaboration avec d'autres arts, par exemple danse, théâtre ou arts visuels.

\section{Planificatrices (prévoir les caractéristiques musicales de l'improvisation) (3)}

Poser des contraintes de départ $(1,2,3)$

Déterminer, avant l'improvisation, certaines de ses caractéristiques musicales, par

exemple un mode, un thème, une structure harmonique, une couleur ou une sonorité de départ.

Improviser sur une structure harmonique (3)

Analyser la structure harmonique d'une pièce provenant du répertoire ; sélectionner un enchaînement harmonique cohérent et intéressant et improviser sur cet enchaînement harmonique ; permet de créer un cadre à la fois rassurant et pertinent pour l'apprenant.

\section{Performatives}

Feindre l'improvisation, pour faire place à l'improvisation ${ }^{\S}$ (2)

Jouer des idées musicales connues en leur donnant un caractère improvisé, par exemple en hésitant, en modulant subtilement la pulsation ou en introduisant des ornements ; permet de se plonger dans un état favorable à la création d'idées authentiquement nouvelles.
Savoirs, savoir-faire et

savoir-être

SF : Audiation

SE : Autonomie et

autodidaxie

SF : Audiation

$\mathrm{S}$ : Motiviques et

thématiques

SF : Audiation

$\mathrm{SF}$ : Développement

$\mathrm{S}$ : Motiviques et

thématiques

SF : Développement

SE : Prise de risques

$\mathrm{S}:$ Rythmiques

S : Timbrals

SF : Maîtrise technique

SE : Prise de risques

SE : Prise de risques

SF : Pré-planification

$\mathrm{S}$ : Tous les savoirs peuvent être ciblés

$\mathrm{S}:$ Harmoniques

$\S \quad$ Souvent l'improvisation n'est pas entièrement créée sur l'inspiration du moment : elle est le résultat d'une combinaison originale d'idées connues et d'idées inventées in situ. 
Focaliser sur le timbre (2) ou sur les textures sonores (3)

Permet notamment d'éviter de penser en termes musicaux plus rationnels (par exemple, la hauteur des notes ou structure harmonique), ce qui favorise l'exploration de nouvelles avenues.

Faire de l'improvisation rythmique pure (3)

Improviser une partie purement rythmique, par exemple en utilisant des percussions corporelles, des instruments de percussion ou des sons sans hauteur à l'instrument. Il s'agit d'une bonne façon d'aborder l'improvisation, qui permet à la fois de simplifier la tâche à l'apprenant et de l'habituer à improviser sans avoir la "peur de la fausse note ", qui est souvent transmise par la pédagogie classique traditionnelle.

Faire de l'improvisation mélodique (3)

Improviser une ligne mélodique, sur une pédale, un ostinato, une métrique ou une structure formelle.

S'ancrer sur tonique-dominante (3)

Improviser en achevant chaque phrase sur la tonique ou la dominante permet de créer une improvisation simple et cohérente.

Continuer à jouer malgré une panne d'inspiration (2)

Répéter délibérément, plus longtemps que le sens esthétique ne le suggère, une note, un motif ou une idée musicale pour " gagner du temps », en attendant que l'inspiration surgisse.

Utilisation créative de l'inattendu (3)

Accepter les « erreurs » ou les « fausses » notes et leur attribuer rétrospectivement de la cohérence musicale, par exemple en les répétant, en les résolvant, en modulant en conséquence ou en les intégrant à une séquence.

Improviser en "explorant le terrain" (2)

Commencer l'improvisation sur une seule note, puis intégrer les hauteurs les unes après les autres, progressivement, en expérimentant diverses possibilités si nécessaire. On pourra s'imaginer mentalement une « carte » tonale ou modale sur l'instrument : les notes deviendront « rouges » ou "vertes", selon qu'elles peuvent être jouées ou pas. Des notes « jaunes », de passage, pourront éventuellement aussi être intégrées à cette carte mentale.

Prioriser la pulsation (2)

Maintenir une pulsation constante quoiqu'il arrive, malgré les éventuelles « fausses notes ", évènements inattendus ou pannes d'inspirations.

Adopter un tempo confortable (2)

Utiliser un temps modéré (autour de 70-80) permettant en règle générale d'utiliser une grande variété de figures rythmiques.

Utiliser des notes brèves (2)

Utiliser des notes brèves, encadrées par deux notes consonantes plus longues, permet d'expérimenter l'effet de diverses hauteurs dans un contexte musical donné. Comme ces notes ne résonnent pas longtemps, elles ne sonneront pas trop « faux » pour l'auditeur.

Utiliser la banque d'idées $(2,3)$

Utiliser des motifs, phrases ou thèmes entreposés dans la boîte d'idées lors de l'improvisation, en tout ou en partie (généralement, si les idées sont tronquées, on en retiendra seulement la «tête », c'est-à-dire les premières notes).

Déployer les éléments techniques (2)

Permuter les éléments techniques pré-mémorisés afin de créer de nouvelles idées
SE : Prise de risques

S : Timbrals

SF : Désapprentissage

$S$ : Rythmiques

\section{$\mathrm{S}:$ Rythmiques}

S : Mélodiques

$\mathrm{S}:$ Harmoniques

$\mathrm{S}$ : Mélodiques

$\mathrm{SE}:$ Prise de risques

$\mathrm{SF}$ : Adéquation

technique

SE : Prise de risques

$\mathrm{SF}$ : Adéquation

technique

SF : Adéquation

technique

S : Mélodiques

SF : Adéquation

technique

$S$ : Rythmiques

$\mathrm{S}:$ Rythmiques

S : Rythmiques

$\mathrm{S}:$ Mélodiques

SF : Banque d'idées

$\mathrm{S}$ : Mélodiques

SF : Banque d'idées

$\mathrm{SF}:$ Développement 
Propositions pédagogiques

Savoirs, savoir-faire et

savoir-être

musicales en temps réel, permet de maximiser le potentiel des (très souvent nombreuses)

heures de répétitions dédiées au perfectionnement technique.

Imiter un instrument (2)

Tenter de reproduire le timbre, l'attaque ou le jeu d'un autre instrument.

Imiter un compositeur, une ceuvre ou un style $(2,3)$

Improviser dans le style d'un compositeur, d'une œuvre ou d'une époque.

Créer une ambiance sonore (2).

Par exemple, mettre en sons un paysage, un évènement ou une émotion.

Faire de l'improvisation "sans style " (3)

Improviser sans s'imposer de limites stylistiques pour laisser jaillir la musique sans restriction.

Faire de l'improvisation générative (3)

Improviser une note à la fois, laisser chaque son tracer la voie au suivant.

Ornementer (3)

Jouer une pièce composée en improvisant les ornements.

Eviter les sentiers battus (2)

Lors de l'improvisation, éviter les « voicings » d'accords, les doigtés, les tonalités, les positions, etc. qui sont bien connus ou automatisés. Par exemple, utiliser les harmonies quartales (2), la gamme chromatique $(2,3)$ ou encore éviter d'installer un centre tonal (2).

Développer ses idées musicales $(2,3)$

Ornementer, varier, répéter, séquencer le matériel musical provenant de la boîte d'idées ou joué précédemment au cours de l'improvisation. Il s'agit d'une stratégie qui permet d'assurer la cohésion de l'improvisation, tout en évitant la monotonie.

Ériger la structure en temps réel $(2,3)$

Créer, en temps réel, la structure de l'improvisation. Par exemple, créer un conséquent, un sommet, des mouvements de tension-détente, des sections contrastées, un pont entre deux sections de l'improvisation ou une progression harmonique.

Faire appel aux techniques contrapuntiques (3)

Par exemple, l'imitation (exacte ou non), le canon ou l'ajout de voix contrapuntiques.

Utiliser les techniques étendues de l'instrument (2)

Explorer ou utiliser les techniques étendues propres à chaque instrument (telles que le tapping, les percussions, les harmoniques ou les microtons).

Établir un contact visuel avec l'instrument en vue de faciliter l'audiation (2)

Regarder l'instrument en jouant peut aider à conscientiser la relation entre la technique de jeu (doigté, position, etc.) employée et la sonorité produite.

Simplifier une idée pour être en mesure de réaliser techniquement en temps réel (2)

Faire, lorsque nécessaire, des compromis sur le plan de la virtuosité en modifiant les idées musicales qui se situent aux limites de ses possibilités techniques, afin de pouvoir les réaliser en temps réel.

SF : Maîtrise technique

S : Timbrals

S : Stylistiques

$\mathrm{S}$ : Atmosphériques

SE : Prise de risques

$\mathrm{S}$ : Stylistiques

SE : Prise de risques

$\mathrm{S}:$ Stylistiques

SF : Développement

$\mathrm{SE}:$ Prise de risques

$\mathrm{S}$ : Rythmiques,

mélodiques,

harmoniques ou tonals

SF : Développement

$\mathrm{S}$ : Rythmiques,

mélodiques ou tonals

$\mathrm{SE}:$ Interactions

$\mathrm{S}$ : Formels

SF : Développement

$\mathrm{S}$ : Contrapuntiques

SF : Maîtrise technique

S : Timbrals

SF : Audiation

SF : Adéquation

technique 